\title{
Hexokinase 2 is a determinant of neuroblastoma metastasis
}

\author{
Liat Edry Botzer ${ }^{1,2}$, Shelly Maman ${ }^{1,2}$, Orit Sagi-Assif ${ }^{1}$, Tsipi Meshel ${ }^{1}$, Ido Nevo ${ }^{1}$, Ilana Yron ${ }^{1}$ and Isaac P Witz ${ }^{*}, 1$ \\ ${ }^{1}$ Department of Cell Research and Immunology, George S. Wise Faculty of Life Sciences, Tel Aviv University, Tel Aviv 69978, Israel
}

\begin{abstract}
Background: Intersecting a genome-wide expression profile of metastatic and nonmetastatic human neuroblastoma xenograft variants with expression profiles of tumours from stage 1 and 4 neuroblastoma patients, we previously characterised hexokinase 2 (HK2) as a gene whose expression was upregulated in both metastatic neuroblastoma variants and tumours from stage 4 neuroblastoma patients.
\end{abstract}

Methods: Local and metastatic neuroblastoma cell variants as well as metastatic neuroblastoma cells genetically manipulated to downregulate the expression of HK2 were utilised for in vitro and in vivo examinations of the involvement of HK2 in neuroblastoma.

Results: Hexokinase 2 expression and its activity levels were increased in neuroblastoma metastatic variants as compared with the local variants. The upregulation of HK2 confers upon the metastatic cells high resistance to the antiproliferative effect of the HK2 inhibitor 3-BrPa and to the chemotherapy agent Deferoxamine. The inhibition of HK2 transcript lowered the proliferation and motility of sh-HK2 cells as compared with sh-control cells. Mice that were inoculated with sh-HK2 cells had a lower incidence of local tumours, smaller tumour volumes and a diminished load of lung metastasis compared with mice inoculated with sh-control cells.

Conclusions: Hexokinase 2 plays a significant role in shaping the malignant phenotype of neuroblastoma and influences the progression of this disease.

Although increased understanding of the metastatic process was achieved in recent years (Gupta and Massague, 2006; Mazzocca and Carloni, 2009; Sethi and Kang, 2011; Maman and Witz, 2013; Klein-Goldberg et al, 2014), the mechanisms underlying the progression of cancer cells to form metastasis are awaiting complete elucidation.

In neuroblastoma, the most common extracranial solid tumour in children, more than half of the patients present with metastatic disease at diagnosis (Maris et al, 2007; Mullassery et al, 2009; Modak and Cheung, 2010). Prevention strategies for neuroblastoma metastasis could be developed if molecules involved in this process are identified and their precise role deciphered.

For the identification of molecular pathways involved in neuroblastoma metastasis, we developed an orthotopic mouse model for human neuroblastoma metastasis (Nevo et al, 2008). An orthotopic implantation of two human neuroblastoma cell lines, MHH-NB11 (Pietsch et al, 1988) and SH-SY5Y (Biedler et al, 1978), into the adrenal gland of athymic nude mice yielded local adrenal tumours as well as lung metastases. After repeated cycles of in vivo passages, local adrenal and lung metastatic variants were generated. Originating in the same human neuroblastoma cell lines, these variants share a common genetic background. Proteomic and transcriptomic differences between these variants may thus be ascribed to their differential malignant phenotype.

Aiming to identify molecular correlates of neuroblastoma metastasis, the genetically identical local and lung metastasising human neuroblastoma variants were subjected to genome-wide expression profiling (Nevo et al, 2010) using a neuroblastomaspecific oligonucleotide microarray (Oberthuer et al, 2006). Genes that were found to be differentially expressed between local and

*Correspondence: Professor IP Witz; E-mail: isaacw@tauex.tau.ac.il

${ }^{2}$ These authors contributed equally to this work.

Received 4 December 2015; revised 11 January 2016; accepted 13 January 2016; published online 17 March 2016

(c) 2016 Cancer Research UK. All rights reserved 0007 - 0920/16 
metastatic neuroblastoma variants were intersected with genes differentially expressed in primary tumours of stage 1 and stage 4 neuroblastoma patients. A neuroblastoma metastatic gene expression signature was identified. This signature included several genes that could serve as therapeutic targets or prognosis markers in this disease (Nevo et al, 2010).

One of the genes that was found to be upregulated in the neuroblastoma metastatic variants compared with the local variants as well as in stage 4 neuroblastoma patients compared with stage 1 patients was hexokinase 2 (HK2) (Nevo et al, 2010). HK2 catalyses the essentially irreversible first step of the glycolytic pathway in which glucose is phosphorylated to glucose-6phosphate (G6P) via phosphate transfer from ATP (Mathupala et al, 2009). In cancer, HK2 is a pivotal player in the 'Warburg effect', a high rate of aerobic glycolysis followed by lactic acid fermentation occurring in malignant tumours (Warburg, 1956).

Glycolytic enzymes including HK2 are overexpressed in at least $70 \%$ of human cancers (Altenberg and Greulich, 2004). Several systemic and cellular mechanisms promote the expression and transcriptional upregulation of $\mathrm{HK} 2$ in malignant tumours including epigenetic events (e.g., demethylation) and gene amplification (Mathupala et al, 2009). The proximal promoter of HK2 contains cis-elements for transcription initiation that are regulated by hypoxia-inducible factor- $1 \alpha$ (HIF-1 $\alpha$ ) and by others (Mathupala et al, 1995, 1997; Marin-Hernandez et al, 2009).

The role of $\mathrm{HK} 2$ and glycolysis in the pathogenesis of neuroblastoma is not thoroughly investigated. Levy et al (2010) confirmed recently that treatment with the HK2 inhibitor, 3-bromo-2-oxopropionate-1-propyl ester (3-BrOP), decreased neuroblastoma cell viability by inducing apoptosis in vitro and generated smaller tumours in vivo.

In the present study we determine the role of HK2 in the formation of neuroblastoma metastasis and suggest that the inhibition of HK2 could potentially be used as future treatment for neuroblastoma.

\section{MATERIALS AND METHODS}

Cell culture. Neuroblastoma local adrenal tumour and lung metastatic variants were generated from the parental cell lines MHH-NB-11 (Pietsch et $a l, 1988$ ) and SH-SY5Y (Biedler et al, 1978) using a mouse model for human neuroblastoma metastasis as detailed here (Nevo et $a l, 2008)$ and were maintained in culture as previously described (Nevo et al, 2008).

Authentication of local and metastatic neuroblastoma cells was performed as described here (Maman et al, 2013). All cultures were periodically examined for mycoplasma contamination.

Animals. Male athymic nude mice (BALB/c background) were purchased from Harlan Laboratories Limited (Jerusalem, Israel). Mice were housed and maintained in laminar flow cabinets under specific pathogen-free conditions at the animal quarters of Tel Aviv University (Tel Aviv, Israel) in accordance with current regulations and standards of the Tel Aviv University institutional animal care and use committee (IAUCUC). Mice were regularly checked by a certified veterinarian who is responsible for health monitoring, animal welfare supervision, experimental protocols and procedure revision. The 7-10-week-old mice were used in the in vivo experiments. The in vivo methodology used in these experiments has been reviewed and approved by the Tel Aviv University IAUCUC.

Antibodies. The following anti-human antibodies were used for western blot analysis: anti-HK2 at a dilution of $1: 500$, anti-ERK2 at $1: 1000$ and anti-AKT at $1: 500$ (Santa Cruz Biotechnology, Inc., Santa Cruz, CA, USA), anti-HIF- $1 \alpha$ (R\&D Systems, Inc., Minneapolis, MN, USA) at $1: 1000$ and anti- $\beta$-tubulin (Sigma-
Aldrich, St Louis, MO, USA) at $1: 2000$. Horseradish peroxidaseconjugated goat anti-mouse and rabbit anti-goat antibodies were used according to the manufacturer's instructions (Jackson Immuno Research Laboratories, West Grove, PA, USA).

Western blotting. Neuroblastoma cells were lysed, resolved on SDS-PAGE and transferred onto nitrocellulose membrane as described previously (Edry Botzer et al, 2011). For the detection of target proteins, membranes were incubated with the relevant antibodies and bands were visualised by enhanced chemiluminescence reactions. The amount of the relevant protein in the lanes was estimated by densitometry using Scion Image software (Scion, Frederick, MD, USA).

HK activity assay. The activity of hexokinases (HKs) was determined by spectrophotometric measurement of the formation of $\beta$-nicotinamide adenine dinucleotide (NADH) that is coupled with the oxidation of G6P, the product of glucose phosphorylation by HK (Polakis and Wilson, 1982; Al jamal, 2005). The coupled assay produces $1 \mu \mathrm{mol}$ of $\mathrm{NADH}$ per $\mu \mathrm{mol}$ of D-glucose phosphorylated.

Neuroblastoma cells were lysed as described before (Edry Botzer et al, 2011), diluted in lysis buffer to a volume of $70 \mu \mathrm{l}$ and added in duplicates to a 96-well plate on ice. Reaction buffer $(20 \mathrm{~mm}$ Hepes $\mathrm{pH}=7.2,5 \mathrm{~mm} \mathrm{MgCl} 2,5 \mathrm{~mm} \mathrm{KCl}$ and $10 \mathrm{~mm}$ glucose, freshly supplemented with $1 \mathrm{Uml}^{-1} \mathrm{PK}, 1 \mathrm{U} \mathrm{ml}^{-1} \mathrm{LDH}, 1 \mathrm{~mm}$ ATP, $1 \mathrm{~mm}$ PEP and $0.18 \mathrm{mg} \mathrm{ml}^{-1} \mathrm{NADH}$ ) was added to the wells in a volume of $200 \mu \mathrm{l}$ and the plate was immediately read using an automated microplate reader (SpectraMax 190; Molecular Devices Corp., Sunnyvale, CA, USA) at $25^{\circ} \mathrm{C}, \mathrm{OD}_{340}$. Measurements were taken every $15 \mathrm{~s}$ for $25 \mathrm{~min}$. The HK activity was calculated as follows: $\Delta \mathrm{OD} / \min (\mathrm{V} \max )-\Delta \mathrm{OD} / \min$ blank $\times 1000 \quad($ for $1000 \mu \mathrm{l}) \times$ dilution factor/6.22 (mM extinction coefficient of NADH).

HK2 inhibition. 3-Bromopyruvate (3-BrPa, Sigma-Aldrich) was reconstituted freshly in PBS before use. Neuroblastoma cells were cultured in growth media containing $0,25,50$ or $100 \mu \mathrm{M}$ of $3-\mathrm{BrPa}$ for $2 \mathrm{~h}$. Cell viability was monitored using XTT-based viability assays.

In vitro viability assays. Neuroblastoma cells were seeded $\left(1 \times 10^{5}\right.$ per well) in triplicate in a 96-well tissue culture plate. Viability was monitored using XTT-based viability assay according to the manufacturer's instructions (Biological Industries, Beit Haemek, Israel),

Inducible knockdown of HK2 by sh-RNA. Lentiviral pTRIPz vectors encoding Tet-inducible sh-HK2 (RHS4696) and nonsilencing control (RHS4743) were purchased from Open Biosystems (Thermo Fisher Scientific, Inc., Carlsbad, CA, USA). These plasmids contain RFP cassette downstream of the Tet/ON promoter.

To produce the infectious viruses, $293 \mathrm{~T}$ packaging cell line was co-transfected using calcium phosphate with the following retroviral backbone plasmids: the TRIPz-RFP/sh-HK2 vectors (a pool of three siRNA sequences), the packaging plasmid pCMV $\Delta 8.2$ and the envelope plasmid pVSV-G.

After $48 \mathrm{~h}$ of incubation, the virus particles in the medium were collected and filtrated $(0.45 \mu \mathrm{m}$ filter $)$. Neuroblastoma lung metastatic cells were plated $\left(2 \times 10^{6}\right) 24 \mathrm{~h}$ before infection. The cells were infected in the presence of $1 \mu \mathrm{g} \mathrm{ml}^{-1}$ Polybrene overnight and the virus-containing medium was replaced with fresh medium. After $72 \mathrm{~h}, 1 \mu \mathrm{g} \mathrm{ml}^{-1}$ Puromycin (InvivoGen, San Diego, CA, USA) was added for 7 days in order to stably select infected cell population. After selection, Puromycin was regularly added to the cultures.

The Tet-On mode of sh-HK2 infected cells was activated by the addition of $1 \mu \mathrm{g} \mathrm{ml}^{-1}$ Doxycycline (Sigma-Aldrich). Efficiency of infection was examined by visualising RFP in Leica DMRB Fluorescence Microscope (Leica Biosystems, Nussloch $\mathrm{GmbH}$, 
Germany). Efficacy of sh-HK2 inhibition was verified by western blotting.

Orthotopic inoculation of tumour cells. The Tet-On mode of shHK2 infected cells was activated by the addition of $1 \mu \mathrm{g} \mathrm{ml}^{-1}$ Doxycycline to neuroblastoma cell cultures for 1 week. An orthotopic inoculation to the adrenal gland of athymic nude mice was performed with either sh-HK2 or sh-control cells $\left(1 \times 10^{6} \%\right.$ $50 \mu \mathrm{l}$ ) as detailed here (Nevo et al, 2008). We monitored and verified the development of local tumours in the adrenal gland using an ultrasound imaging in vivo as previously described (Edry Botzer et al, 2011).

For the in vivo activation of sh-HK2 Tet-On cells, $5 \mathrm{mg} \mathrm{ml}^{-1}$ Dox and $1.25 \mathrm{mg} \mathrm{ml}^{-1}$ sucrose were added to the drinking water (replaced every $72 \mathrm{~h}$ ). Two weeks after local tumours were detected by ultrasound, the mice were moribund and killed. Mice inoculated with sh-control cells were killed at the same time point for comparison. Lungs were harvested, immediately put on dry ice and kept in $-80^{\circ} \mathrm{C}$ until use.

Determination of the presence of human cells in mouse tissues by qRT-PCR. The PCR oligonucleotide primers were designed according to sequences derived from the National Center for Biotechnology Information (NCBI) database to react specifically with the human plasminogen activator receptor urokinase type (PLAUR) sequence and have no crossreactivity with the mouse PLAUR sequence (forward: 5'-GTCACCTATTCCCGAAGCCG-3', reverse: $5^{\prime}$ CGGTACTGGACATCCAGGTCT-3').

Amplification reactions were performed with SYBR Green I (Thermo Fisher Scientific, ABgene, Epsom, UK) in triplicate in Rotor-gene 6000TM (Qiagen, Valencia, CA, USA) as previously described (Edry Botzer et al, 2011).

Wound healing assay. The Tet-On mode of sh-HK2-infected cells was activated by the addition of $1 \mu \mathrm{g} \mathrm{ml}^{-1}$ Doxycycline to neuroblastoma cell cultures for 1 week. Cells were then seeded in 6-well plates coated with $10 \mu \mathrm{g} \mathrm{ml}^{-1}$ fibronectin (Biological Industries). Upon confluence, the cell monolayer was wounded with a plastic tip, then washed twice with RPMI medium and replaced with fresh growth medium. Closure of the denuded area was monitored using an inverted microscope (Eclipse TE 2000-S; Nikon, Enfield, CT, USA) fitted with a digital camera (DXM1200F; Nikon). Photos were taken at days 0, 1, 2 and 3 after the wounding. Analysis of wound closure was performed using WIMASIS image analysis software (Wimasis $\mathrm{GmbH}$, Munich, Germany).

Hypoxia assays. Neuroblastoma cells $\left(2 \times 10^{6}\right.$ per well $)$ were seeded in tissue culture plates. After an overnight incubation, the plates were transferred into hypoxia chamber (1\% oxygen) for $4 \mathrm{~h}$. Control cells were left in normoxia conditions. Hypoxia-mimetic conditions were induced by the addition of 25 , and $50 \mu \mathrm{m}$ Deferoxamine mesylate salt (Sigma-Aldrich) to culture plates for $24 \mathrm{~h}$. No Deferoxamine was added to control cells. Nonadherent and adherent cells were lysed in RIPA buffer on ice. The expression of HK2 and HIF- $1 \alpha$ was analysed using western blot analysis.

Statistical analysis. Paired and unpaired Student's $t$-test and Wilcoxon rank test were used to compare results.

\section{RESULTS}

HK2 is highly expressed and activated in metastatic neuroblastoma variants. A gene expression analysis of local (adrenal) and lung metastasising human neuroblastoma cells using a neuroblastoma-specific oligonucleotide microarray (Oberthuer et al, 2006) revealed that $\mathrm{HK} 2$, the enzyme that is responsible for the first step in most glucose metabolism pathways, was upregulated in neuroblastoma lung metastasising variants, as well as in stage 4 neuroblastoma patients as compared with local adrenal variants and stage 1 patients (Nevo et al, 2010). The upregulation of the expression of HK2 in metastatic neuroblastoma variants compared with its expression in the local (adrenal) variants was observed at the protein level as well (Nevo et al, 2010). An examination of the enzymatic activity of HK isoforms (Polakis and Wilson, 1982) in local and metastatic neuroblastoma variants indicated that the activity was significantly higher in the metastatic variants than in the corresponding local variants (Figure 1).

Hypoxia induces high levels of HK2 in metastatic neuroblastoma variants. The following experiments were aimed to identify the mechanism that promotes the upregulated expression and the high enzymatic activity of HK2 in neuroblastoma metastases. Hypoxia-inducible factor- $1 \alpha$ (HIF- $1 \alpha)$ promotes the transcription of a broad range of genes involved in cancer progression and metastasis (Haase, 2010). One of these genes is HK2, harbouring consensus motifs for HIF- $1 \alpha$ in its promoter (Mathupala et al, 2001). We previously demonstrated that hypoxia-mimetic conditions (Deferoxamine treatment) upregulate HIF- $1 \alpha$ expression in metastatic neuroblastoma variants to significantly higher levels than in local neuroblastoma variants exposed to the same conditions (Nevo et al, 2008).

We asked whether the high HK2 expression in the metastatic variants is because of the high activation of HIF- $1 \alpha$ in these cells, and whether HK2 is regulated by hypoxic conditions. To answer these questions we examined the expression levels of these molecules under normoxia and hypoxia as well as by mimicking hypoxic conditions using Deferoxamine.

Cells were treated with 0,25 and $50 \mu \mathrm{M}$ Deferoxamine or kept under hypoxic conditions ( $1 \%$ oxygen) in a hypoxia chamber. The expression levels of HIF- $1 \alpha$ and HK2 were measured by western blot analysis. As demonstrated in Figure 2, under normoxia HIF$1 \alpha$ expression was undetectable in the local and metastatic variants. Hypoxia and Deferoxamine treatment induced the expression of HIF- $1 \alpha$ in all neuroblastoma variants. An upregulation of HK2 expression correlated with the upregulation of HIF- $1 \alpha$ under hypoxic and hypoxia-mimetic conditions and was higher in the metastatic variants than in the corresponding local variants (Figure 2).

We suggest that the high expression and enzymatic activity level of HK2 in the metastatic variants are a result of the selective pressure exerted by hypoxic conditions that enhance the malignant and metastatic phenotype of these cells.

Differential sensitivity of neuroblastoma variants to the HK2 inhibitor 3-BrPa. The pyruvate analogue $3-\mathrm{BrPa}$ is an inhibitor of $\mathrm{HK} 2$ that reduces the metabolism of cancer cell energy and triggers cell death (Chen et al, 2009). We asked whether the higher

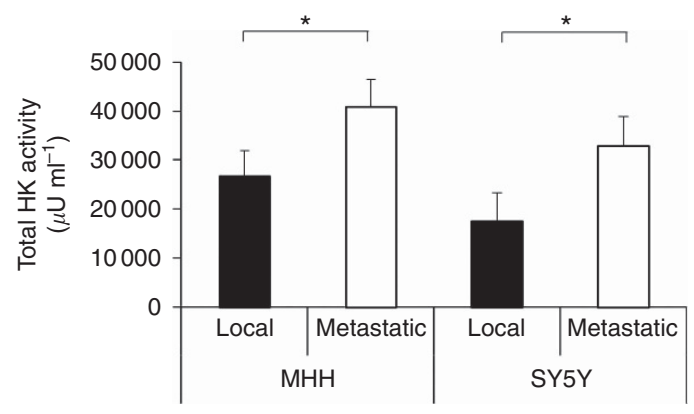

Figure 1. Activity levels of HK2 are higher in neuroblastoma metastatic variants than in local variants. Total HK activity in neuroblastoma variants was determined by measuring microunit enzyme per $\mathrm{ml}\left(\mu \cup \mathrm{ml}^{-1}\right)$. Data are means of three independent experiments + s.d. Significance was evaluated using Student's t-test. $\star P<0.05$. 
A

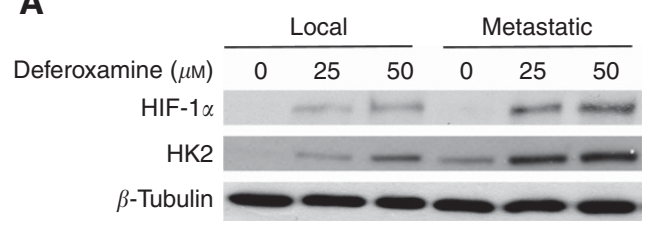

B
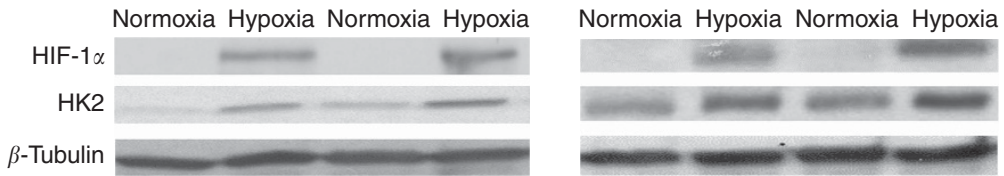

Figure 2. Hypoxic conditions induce high levels of HK2 expression in neuroblastoma metastatic variants. Local and metastatic neuroblastoma variants were treated with 0, 25 and $50 \mu \mathrm{m}$ Deferoxamine (A) or incubated in a hypoxia chamber (1\% oxygen) (B). Following treatment, the expression level of HIF-1 $\alpha$ and HK2 was measured by western blot analysis. Shown are the results of one representative blot out of four independent experiments.

A

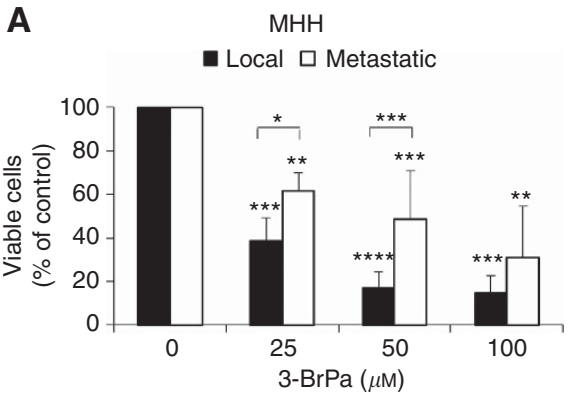

SY5Y

- Local $\square$ Metastatic

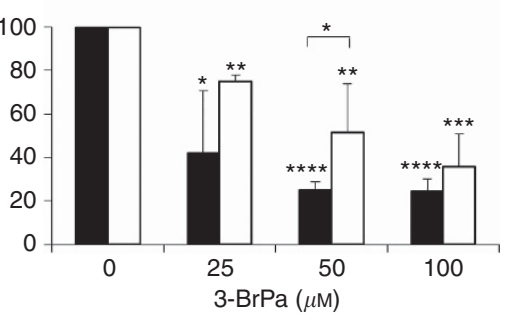

B

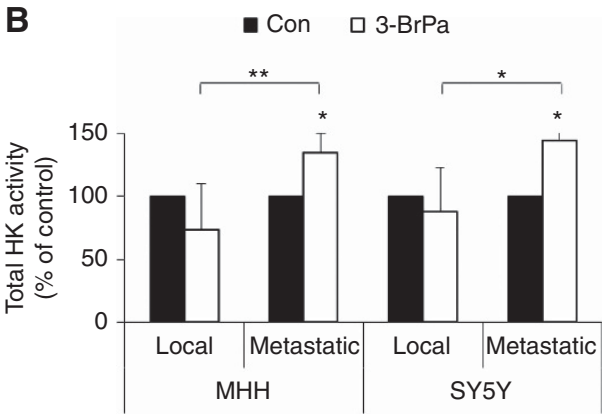

Figure 3. Metastatic neuroblastoma variants are more resistant than local variants to growth inhibition mediated by the HK2 inhibitor 3-BrPa. (A) Viability of local and metastatic neuroblastoma variants was determined by XTT-based viability assays following treatment with 25,50 and $100 \mu \mathrm{m} 3-\mathrm{BrPa}$. (B) The activity of all HK isoforms in neuroblastoma variants was determined following treatment with $50 \mu \mathrm{m} 3-\mathrm{BrPa}$ by measuring microunit enzyme per $\mathrm{ml}\left(\mu \cup \mathrm{ml}^{-1}\right)$ normalised to control untreated cells. Data are means of three independent experiments + s.d. Significance was evaluated using Student's t-test. ${ }^{\star} P<0.05,{ }^{\star \star} P<0.01,{ }^{\star \star \star} P<0.005$ and ${ }^{\star \star \star \star *} P<0.001$.

expression and activity levels of HK2 in the metastatic variants than in the local variants affects the sensitivity of these cells to $3-\mathrm{BrPa}$.

Neuroblastoma cells were treated with $0,25,50$ and $100 \mu \mathrm{M} 3-$ $\mathrm{BrPa}$ for $2 \mathrm{~h}$. Cell viability was then measured using XTT-based proliferation assays. Figure $3 \mathrm{~A}$ demonstrates that the viability of all 3-BrPa-treated neuroblastoma variants was impaired. However, the metastatic variants of both neuroblastoma tumours $(\mathrm{MHH}$ and SY5Y) were more resistant to the viability restraining effects of $3-\mathrm{BrPa}$ than the local variants, possibly because of higher reservoir levels of HK2 in the metastatic cells. To establish whether 3- $\mathrm{BrPa}$ treatment inhibited HK2 activity, we measured the enzymatic activity of all $\mathrm{HK}$ isoforms in cells treated with $50 \mu \mathrm{M}$ 3-BrPa. Figure $3 \mathrm{~B}$ demonstrates that $3-\mathrm{BrPa}$ treatment did not influence $\mathrm{HK}$ activity in the local neuroblastoma variants, possibly because of the fact that these variants express low levels of HK2 and therefore blockage with $3-\mathrm{BrPa}$ does not significantly influence total HK activity in these cells. In the treated metastatic variants, total HK activity was increased. It is possible that the inhibition of HK2 in the metastatic cells triggered a compensatory mechanism of other $\mathrm{HK}$ isoforms that eventually raised total $\mathrm{HK}$ activity in these cells.

Inducible downregulation of $\mathrm{HK} 2$ expression in metastatic neuroblastoma variants. In order to examine the possibility that high expression levels and high enzymatic activity of HK2 play a role in conferring a metastatic phenotype upon neuroblastoma cells, we downregulated the expression of $\mathrm{HK} 2$ by Tet-On Tetracycline-controlled transcriptional activation of sh-RNA plasmids specific to human HK2 (sh-HK2) and examined the influence of this downregulation on metastatic neuroblastoma variants. Control cells were infected with scrambled sequence (sh-control). The sh-HK2 and control plasmids contained a red fluorescent protein (RFP) expression cassette downstream of the Tet-On promoter. The infected cells in each experiment were activated with Doxycycline and visualised by fluorescence microscopy to confirm the transcriptional state of the integrated plasmid. In addition, the expression level of HK2 was analysed using immunoblotting.

At 1 week following plasmid activation, the expression level of HK2 in sh-HK2 cells was reduced to $60 \%$ of its expression in sh-control cells (Figure 4A). At 2 weeks post activation, HK2 expression dropped to $10 \%$ of its expression in sh-control cells (Figure 4A). Total HK activity was decreased by $50 \%$ in sh-HK2 cells compared with sh-control cells (Figure 4B). HK activity assays measure the activity of all HK isoforms (Polakis and Wilson, 1982). Therefore, downregulating HK2 in these cells does not completely block total HK activity or the metabolic flux in the cells.

High expression levels of $\mathrm{HK} 2$ account for the resistance of metastatic neuroblastoma cells to $3-\mathbf{B r P a}$. Based on the results reported above that metastatic neuroblastoma cells expressing high levels of HK2 are more resistant to the toxic effects of the HK2 inhibitor $3-\mathrm{BrPa}$ than the local variants expressing lower levels of HK2 enzyme, we hypothesised that resistance to the toxic effects of the inhibitor is because of the high level of endogenous expression of HK2. 


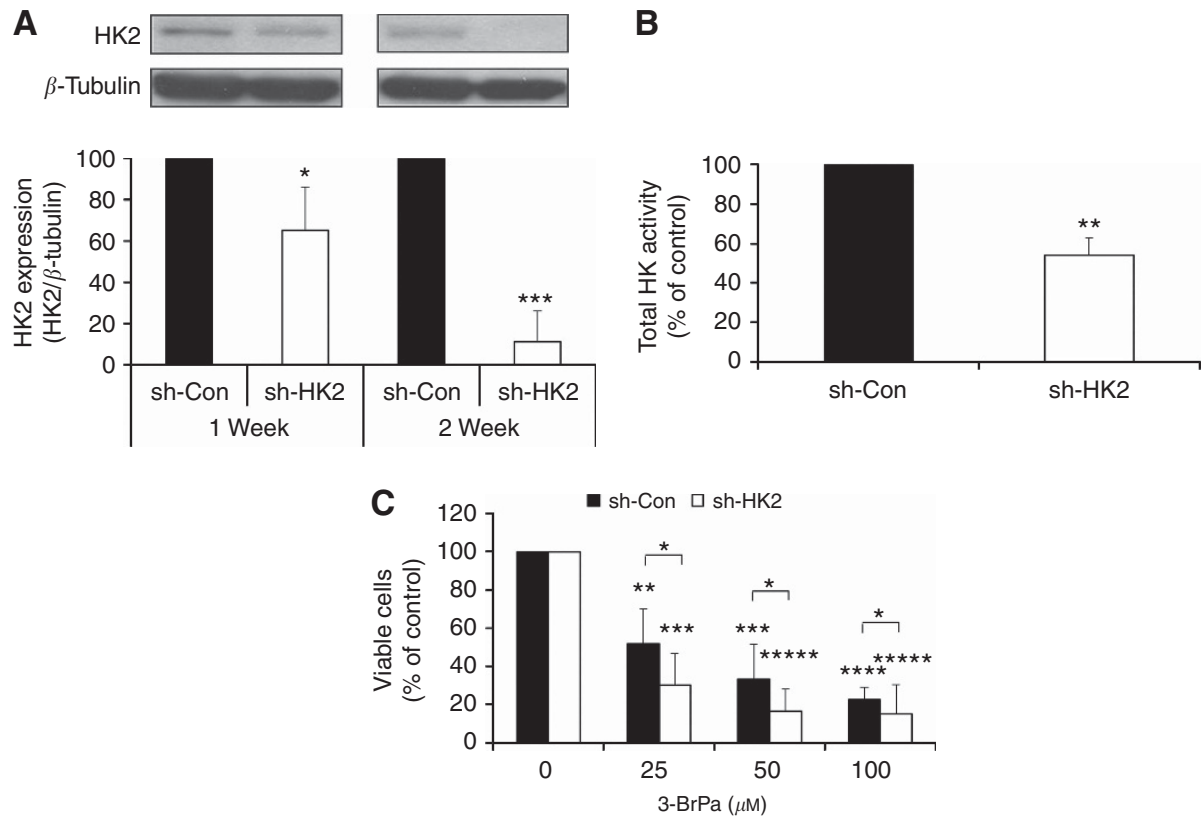

Figure 4. Sh-HK2 reduces HK2 expression level and total HK activity and induces sensitivity to the HK2 inhibitor 3-BrPa. (A) Whole-cell lysates of neuroblastoma sh-HK2 or sh-Con cells were subjected to western blot analysis and immunostaining. The expression level was measured 1 week or 2 weeks following plasmid activation. (B) Total HK activity in neuroblastoma sh-Con or sh-HK2 cells was determined by measuring microunit enzyme per $\mathrm{ml}\left(\mu \cup \mathrm{ml}^{-1}\right)$. (C) Viability of sh-HK2 and sh-Con cells was determined by XTT-based viability assay following treatment with 25,50 and $100 \mu \mathrm{m} 3-\mathrm{BrPa}$. Data are means of three independent experiments + s.d. The blots shown are of a representative experiment out of three. Significance was evaluated using Student's t-test. ${ }^{\star} P<0.05$, ${ }^{\star \star} P<0.01$, ${ }^{\star \star \star} P<0.005$, ${ }^{\star \star \star \star} P<0.001$ and ${ }^{\star \star \star \star \star *} P<0.0005$.

Figure 4C shows that indeed the sensitivity of sh-HK2 cells to $3-\mathrm{BrPa}$ is significantly higher than that of sh-control cells. These results support the hypothesis that the low sensitivity of the metastatic variants to $3-\mathrm{BrPa}$ is because of high expression levels of HK2 in these variants.

Knocking down HK2 expression ameliorates the malignant phenotype of metastatic neuroblastoma cells. Metastatic and local human neuroblastoma variants exhibit a differential malignant phenotype (Nevo et al, 2008). The metastatic variants are highly tumourigenic in xenografted nude mice, yield a higher metastatic load in these mice, possess a higher migratory capacity and are relatively more resistant to chemotherapy than the corresponding local variants (Nevo et al, 2008).

If high levels of $\mathrm{HK} 2$ are related to a high malignancy phenotype, it is to be expected that downregulating the levels of this enzyme would ameliorate the malignancy phenotype of the cells. In the in vitro and in vivo experiments described below we examined this hypothesis.

Proliferation and morphology. HK2 promotes tumour cell growth and survival (Pedersen et al, 2002). In order to evaluate the effects of silencing HK2 expression on neuroblastoma cell proliferation, we measured the viability of the infected cells after plasmid activation using XTT-based viability assays. No influence on cell viability was apparent in sh-control and sh-HK2 cells at $96 \mathrm{~h}$ (Figure 5A) and up to 7 days (Figure 5B) after plasmid activation. However, the viability of sh-HK2 cells cultured for 14 and 17 days after plasmid activation was dramatically decreased (Figure 5B).

Another apparent difference between sh-control and sh-HK2 cells was their morphology. sh-HK2 cells grew as crowded clumps whereas sh-control cells presented with an elongated shape and a higher degree of spreading associated with the morphology of the lung metastatic variants from which they originated (Figure 5B). Thus, downregulating the expression of HK2 resulted in a changed morphology of the metastatic variant to resemble that of the less malignant local tumour variant (Nevo et al, 2008).

Migratory capacity. Little is known about the involvement of HK2 in the migration of tumour cells. Migratory capacity of sh-HK2 neuroblastoma cells was examined in wound healing assays and analysed by WIMASIS software. Figure 5C shows that downregulation of HK2 reduces motility. The sh-control cells closed the wound more rapidly than sh-HK2 cells (57\% and 25\% closure, respectively, $24 \mathrm{~h}$ after wounding). At the end of the experiment, sh-control cells closed $100 \%$ of the wound, whereas sh-hk 2 cells closed only $30 \%$ of it.

These results suggest that HK2 is indeed involved in the metastatic process, of which migratory capacity of cancer cells is a key factor. This suggestion is supported by our previous observation that neuroblastoma metastatic variants expressing high levels of HK2 migrate faster than nonmetastatic local variants that express low levels of HK2 (Nevo et al, 2008).

Tumourigenicity and metastasis formation. The aim of the next set of experiments was to determine the capacity of sh-HK2 and sh-control cells to form local tumours in the adrenal gland and to generate distant metastases following an orthotopic xenograft of the cells into nude mice.

The sh-RNA plasmids were activated in vitro a week before the inoculation and maintained activated in vivo by adding Doxycycline to the drinking water of the mice. Local tumour incidence and volume was determined using ultrasound (US) 6 weeks post inoculation. The presence of metastatic cells in the lungs was determined by measuring the amount of human RNA in the lungs of the xenografted mice using qRT-PCR.

Mice inoculated with sh-HK2 cells had a lower incidence of local adrenal tumours (Figure 5D) and smaller tumour volumes (Figure 5E). The load of lung metastasis was notably lower in mice inoculated with sh-HK2 cells than in mice inoculated with shcontrol cells (Figure 5F). These results were statistically significant as determined by Wilcoxon rank test $(P<0.05)$. These results 


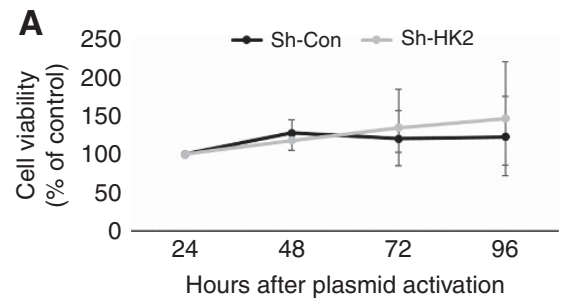

B
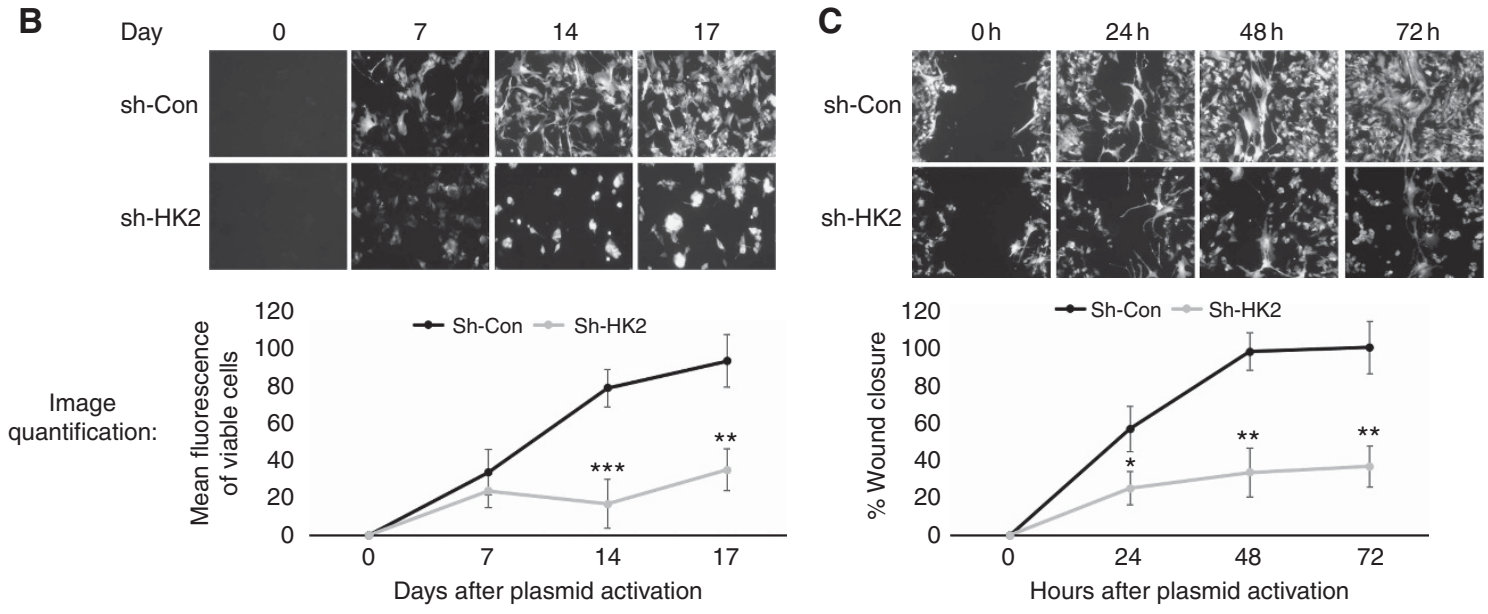

D

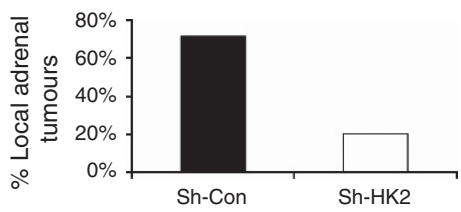

E

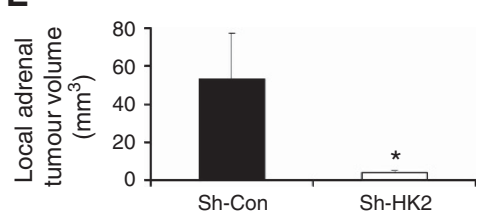

$\mathbf{F}$

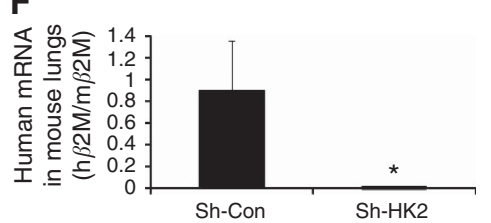

Figure 5. Downregulation of HK2 expression reduces the malignant phenotype of neuroblastoma cells. (A) Sh-Con and Sh-HK2 cell viability was examined using XTT-based viability assays 24,48 and $72 \mathrm{~h}$ post plasmid activation. (B) Sh-Con and sh-HK2 cells were activated (day 0 ) and fluorescent pictures demonstrating cell proliferation and morphology were taken on days $0,7,14$ and 17 post plasmid activation. (C) Fluorescence photomicrographs of scratched monolayers in a wound healing assay of sh-Con and sh-HK2 cells. The scratches were performed 1 week after plasmid activation $(0 \mathrm{~h})$. Pictures were taken at 0, 24, 48 and $72 \mathrm{~h}$ after the scratch was performed. (D) Percentage of the development of local adrenal tumours in mice inoculated with sh-Con and sh-HK2 cells. (E) Volume measurements of local adrenal tumours in mice inoculated with sh-Con and sh-HK2 cells. (F) Quantification of human mRNA in mouse lungs using qRT-PCR reactions indicating lung metastasis of human origin in mice inoculated with sh-Con and sh-HK2 cells. Data are means of three independent experiments + s.d. (A-C) or of mice in each group $(n=14,7$ mice in each group) + s.d. (D-F). Significance was evaluated using Student's t-test $(\mathbf{A}-\mathbf{C})$ or Wilcoxon rank test $(\mathbf{E}$ and $\mathbf{F}) .{ }^{\star} P<0.05,{ }^{\star \star} P>0.01$ and $\star \star \star P<0.005$.

clearly indicate that HK2 is functionally involved in tumourigenesis and metastasis of neuroblastoma cells.

\section{DISCUSSION}

This study provides information on molecular mechanisms in human neuroblastoma that links three cancer hallmarks (Hanahan and Weinberg, 2011): increased viability (proliferative capacity and/or cell death resistance), deregulated cellular metabolism and metastasis.

Several genes that regulate cancer spread and dissemination and those that contribute to organ-specific metastasis have been identified in different cancer types such as lung and bone metastasis in breast cancer (Chambers et al, 2002; Nguyen et al, 2009). However, little is known about the molecules involved in neuroblastoma metastasis.

In a previous study, we described the development of an in vivo model for neuroblastoma metastasis (Nevo et al, 2008). Using a neuroblastoma-specific oligonucleotide microarray (Oberthuer et al, 2006), we identified genes that were differentially expressed in local and metastatic variants originating in the same neuroblastoma tumours (Nevo et al, 2010). Comparing the transcriptomes of local and metastatic variants and intersecting them with the transcriptomes of cells from local tumours of phase 1 and 4 neuroblastoma patients, we were able to identify a small, clinically relevant, gene set associated with neuroblastoma metastatic progression (Nevo et al, 2010).

Hexokinase 2, the enzyme that catalyses the first committed step of glucose metabolism, was identified as a member of this gene set, exhibiting a significantly higher expression level in metastatic neuroblastoma variants as well as in tumours derived from stage 4 neuroblastoma patients than in local neuroblastoma variants and in tumours derived from stage 1 patients. In the present study, we confirmed this differential expression and showed that high HK2 expression levels confer a more malignant, tumourigenic and metastatic phenotype on neuroblastoma cells.

Hexokinase 2 plays a pivotal and direct role in the Warburg effect, a hallmark of many malignant tumours. Hexokinase 2 is usually expressed in very low levels in normal cells (Pedersen et al, 2002), whereas in many tumours, for example, liver (Stubbs et al, 2003; Marin-Hernandez et al, 2006), breast (Balinsky et al, 1984) and cervical cancer (Marin-Hernandez et al, 2006), as well as 
pancreatic and colon carcinomas (Peng et al, 2008a, 2009), HK2 expression is extensively increased, consistent with enhanced glycolytic activity. Additional findings support the possible involvement of $\mathrm{HK} 2$ in the metastatic cascade. For example, HK2 overexpression contributes to progression of hepatocellular carcinoma (Peng et al, 2008b) and of cholangiocellular carcinoma (Paudyal et al, 2008). Despite these reports and despite the fact that HK2 is used for the detection of metastasis by positron emission tomography (PET), there are only a few studies examining its role in metastasis formation.

Hypoxia is a common characteristic of the solid tumour microenvironment (Fardin et al, 2010) including rapidly expanding neuroblastoma tumours. The HIF- $1 \alpha$ is a metastasis-regulating gene strongly linked to invasion, tumourigenesis and angiogenesis (Subarsky and Hill, 2003; Brahimi-Horn and Pouyssegur, 2006). The observed correlation between HK2 expression and HIF- $1 \alpha$ expression implies that one of the mechanisms that promote the upregulated expression activity levels of HK2 in neuroblastoma metastases is coupled with low oxygen levels associated with solid tumours. Interestingly, the upregulation of HIF- $1 \alpha$ and HK2 expression under hypoxic and hypoxia-mimetic conditions was more significant in the metastatic variants than in the local ones. This suggests that the metastatic variants cope more efficiently than local variants with stressful hypoxic microenvironments.

Glycolytic inhibitions have been proposed as a therapeutic strategy for cancer treatment (Sheng et al, 2009; Pradelli et al, 2010). $3-\mathrm{BrPa}$ is a small-molecule analogue of pyruvate that inhibits HK2 and prevents glucose from entering the glycolytic pathway (Ko et al, 2001). In HCC (Kim et al, 2007; Vali et al, 2007), breast cancer (Buijs et al, 2009), melanoma (Qin et al, 2010) and colon cancer (Sanchez-Arago and Cuezva, 2011), 3-BrPa exhibits promising anticancer activities.

Treatment with $3-\mathrm{BrPa}$ reduced the $\mathrm{HK}$ activity and the viability of both local and metastatic neuroblastoma variants. However, the metastatic variants, expressing higher levels of HK2, were significantly less influenced by this treatment - their viability was only slightly impaired and their total HK activity even increased under this treatment.

Two nonmutually exclusive mechanisms may account for the differential sensitivity of the local and metastatic variants to 3$\mathrm{BrPa}$. The first is resistance of the metastatic cells to $3-\mathrm{BrPa}$ that results from high reserves of active HK2 enzyme that could allow these cells to maintain a high HK2 activity even under the inhibitory influence of $3-\mathrm{BrPa}$. The second mechanism that could explain this phenomenon is that the metastatic variants upregulate other isoforms of $\mathrm{HK}$ as a compensation mechanism in order to maintain a high glycolytic rate when HK2 activity is inhibited by 3 $\mathrm{BrPa}$. If indeed such a compensatory mechanism exists, a treatment for neuroblastoma should inhibit the activity of all HK isoforms.

The efficient inhibition of HK2 by sh-RNA in neuroblastoma metastatic variants lowered the malignant phenotype of the cells. The sh-HK2 cells exhibited a lower proliferation rate and migratory capacity and generated a lower incidence of local adrenal tumours, a smaller tumour volume and a significantly lower lung metastasis rate.

Based on the results of this study we propose that HK2, playing a key role in the progression of neuroblastoma, may serve as a therapeutic target for this disease.

\section{ACKNOWLEDGEMENTS}

We thank Dr Naam Kariv and Dr Mickey Harlev from the Animal Care Facilities, Sackler Faculty of Medicine, Tel-Aviv University, for all the help with animal experiments. We also thank Mrs Dafna Shryber for the Ultrasound imaging. We thank the German Research Foundation (Deutche Forschungsgemeinschaft (DFG), Grant BA4027/6-1), the James \& Rita Leibman Endowment Fund for Cancer Research for their financial support, The Fred August and Adele Wolpers Charitable Fund (Clifton, NJ, USA) and the Sara and Natan Blutinger Foundation (West Orange, NJ, USA).

\section{CONFLICT OF INTEREST}

The authors declare no conflict of interest.

\section{REFERENCES}

Al jamal JA (2005) Involvement of porin N,N-dicyclohexylcarbodiimidereactive domain in hexokinase binding to the outer mitochondrial membrane. Protein J 24(1): 1-8.

Altenberg B, Greulich KO (2004) Genes of glycolysis are ubiquitously overexpressed in 24 cancer classes. Genomics 84(6): 1014-1020.

Balinsky D, Platz CE, Lewis JW (1984) Enzyme activities in normal, dysplastic, and cancerous human breast tissues. J Natl Cancer Inst 72(2): 217-224.

Biedler JL, Roffler-Tarlov S, Schachner M, Freedman LS (1978) Multiple neurotransmitter synthesis by human neuroblastoma cell lines and clones. Cancer Res 38(11 Pt 1): 3751-3757.

Brahimi-Horn C, Pouyssegur J (2006) The role of the hypoxia-inducible factor in tumor metabolism growth and invasion. Bull Cancer 93(8): E73-E80.

Buijs M, Vossen JA, Geschwind JFH, Ishimori T, Engles JM, Acha-Ngwodo O, Wahl RL, Vali M (2009) Specificity of the anti-glycolytic activity of 3-bromopyruvate confirmed by FDG uptake in a rat model of breast cancer. Invest New Drug 27(2): 120-123.

Chambers AF, Groom AC, MacDonald IC (2002) Dissemination and growth of cancer cells in metastatic sites. Nat Rev Cancer 2(8): 563-572.

Chen Z, Zhang H, Lu WQ, Huang P (2009) Role of mitochondria-associated hexokinase II in cancer cell death induced by 3-bromopyruvate. Biochim Biophys Acta 1787(5): 553-560.

Edry Botzer L, Maman S, Sagi-Assif O, Meshel T, Nevo I, Bäuerle T, Yron I, Witz IP (2011) Lung-residing metastatic and dormant neuroblastoma cells. Am J Pathol 179(1): 524-536.

Fardin P, Cornero A, Barla A, Mosci S, Acquaviva M, Rosasco L, Gambini C, Verri A, Varesio L (2010) Identification of multiple hypoxia signatures in neuroblastoma cell lines by $1(1)-1(2)$ regularization and data reduction. J Biomed Biotechnol 2010: 878709.

Gupta GP, Massague J (2006) Cancer metastasis: building a framework. Cell 127(4): 679-695.

Haase VH (2010) The sweet side of HIF. Kidney Int 78(1): 10-13.

Hanahan D, Weinberg RA (2011) Hallmarks of cancer: the next generation. Cell 144(5): 646-674.

Kim W, Yoon JH, Jeong JM, Cheon GJ, Lee TS, Yang JI, Park SC, Lee HS (2007) Apoptosis-inducing antitumor efficacy of hexokinase II inhibitor in hepatocellular carcinoma. Mol Cancer Ther 6(9): 2554-2562.

Klein-Goldberg A, Maman S, Witz IP (2014) The role played by the microenvironment in site-specific metastasis. Cancer Lett 352(1): 54-58.

Ko YH, Pedersen PL, Geschwind JF (2001) Glucose catabolism in the rabbit VX2 tumor model for liver cancer: characterization and targeting hexokinase. Cancer Lett 173(1): 83-91.

Levy A, Zage P, Akers L, Ghisoli M, Chen Z, Fang W, Kannan S, Graham T, Zeng L, Franklin A, Huang P, Zweidler-McKay P (2010) The combination of the novel glycolysis inhibitor 3-BrOP and rapamycin is effective against neuroblastoma. Invest New Drug 30: 1-9.

Maman S, Edry-Botzer L, Sagi-Assif O, Meshel T, Yuan W, Lu W, Witz IP (2013) The metastatic microenvironment: lung-derived factors control the viability of neuroblastoma lung metastasis. Int J Cancer 133(10): 2296-2306.

Maman S, Witz IP (2013) The metastatic microenvironment. In The Tumor Immunoenvironment, Shurin MR, Umansky V, Malyguine A (eds). Vol. XVI, pp 15-38. Springer: New York, NY.

Marin-Hernandez A, Gallardo-Perez JC, Ralph SJ, Rodriguez-Enriquez S, Moreno-Sanchez R (2009) HIF-1alpha modulates energy metabolism in cancer cells by inducing over-expression of specific glycolytic isoforms. Mini Rev Med Chem 9(9): 1084-1101. 
Marin-Hernandez A, Rodriguez-Enriquez S, Vital-Gonzalez PA, Flores-Rodriguez FL, Macias-Silva M, Sosa-Garrocho M, Moreno-Sanchez R (2006) Determining and understanding the control of glycolysis in fast-growth tumor cells. Flux control by an over-expressed but strongly product-inhibited hexokinase. FEBS J 273(9): 1975-1988.

Maris JM, Hogarty MD, Bagatell R, Cohn SL (2007) Neuroblastoma. Lancet 369(9579): 2106-2120.

Mathupala SP, Heese C, Pedersen PL (1997) Glucose catabolism in cancer cells. The type II hexokinase promoter contains functionally active response elements for the tumor suppressor p53. J Biol Chem 272(36): 22776-22780.

Mathupala SP, Ko YH, Pedersen PL (2009) Hexokinase-2 bound to mitochondria: cancer's stygian link to the "Warburg Effect" and a pivotal target for effective therapy. Semin Cancer Biol 19(1): 17-24.

Mathupala SP, Rempel A, Pedersen PL (1995) Glucose catabolism in cancer cells. Isolation, sequence, and activity of the promoter for type II hexokinase. J Biol Chem 270(28): 16918-16925.

Mathupala SP, Rempel A, Pedersen PL (2001) Glucose catabolism in cancer cells: identification and characterization of a marked activation response of the type II hexokinase gene to hypoxic conditions. J Biol Chem 276(46): 43407-43412.

Mazzocca A, Carloni V (2009) The metastatic process: methodological advances and pharmacological challenges. Curr Med Chem 16(14): 17041717.

Modak S, Cheung NKV (2010) Neuroblastoma: therapeutic strategies for a clinical enigma. Cancer Treat Rev 36(4): 307-317.

Mullassery D, Dominici C, Jesudason EC, McDowell HP, Losty PD (2009) Neuroblastoma: contemporary management. Arch Dis Child Educ Pract Ed 94(6): 177-185.

Nevo I, Oberthuer A, Botzer E, Sagi-Assif O, Maman S, Pasmanik-Chor M, Kariv N, Fischer M, Yron I, Witz IP (2010) Gene-expression-based analysis of local and metastatic neuroblastoma variants reveals a set of genes associated with tumor progression in neuroblastoma patients. Int J Cancer 126(7): 1570-1581.

Nevo I, Sagi-Assif O, Edry Botzer L, Amar D, Maman S, Kariv N, Leider-Trejo LE, Savelyeva L, Schwab M, Yron I, Witz IP (2008) Generation and characterization of novel local and metastatic human neuroblastoma variants. Neoplasia 10(8): 816-827.

Nguyen DX, Bos PD, Massague J (2009) Metastasis: from dissemination to organ-specific colonization. Nat Rev Cancer 9(4): 274-284.

Oberthuer A, Berthold F, Warnat P, Hero B, Kahlert Y, Spitz R, Ernestus K, Konig R, Haas S, Eils R, Schwab M, Brors B, Westermann F, Fischer M (2006) Customized oligonucleotide microarray gene expression-based classification of neuroblastoma patients outperforms current clinical risk stratification. J Clin Oncol 24(31): 5070-5078.

Paudyal B, Oriuchi N, Paudyal P, Higuchi T, Nakajima T, Endo K (2008) Expression of glucose transporters and hexokinase II in cholangiocellular carcinoma compared using [F-18]-2-fluro-2-deoxy-D-glucose positron emission tomography. Cancer Sci 99(2): 260-266.

Pedersen PL, Mathupala S, Rempel A, Geschwind JF, Ko YH (2002) Mitochondrial bound type II hexokinase: a key player in the growth and survival of many cancers and an ideal prospect for therapeutic intervention. Biochim Biophys Acta 1555(1-3): 14-20.

Peng Q, Zhou J, Zhou Q, Pan F, Zhong D, Liang H (2009) Silencing hexokinase II gene sensitizes human colon cancer cells to 5 -fluorouracil. Hepatogastroenterology 56(90): 355-360.

Peng Q, Zhou Q, Zhou J, Zhong D, Pan F, Liang H (2008a) Stable RNA interference of hexokinase II gene inhibits human colon cancer LoVo cell growth in vitro and in vivo. Cancer Biol Ther 7(7): 1128-1135.

Peng SY, Lai PL, Pan HW, Hsiao LP, Hsu HC (2008b) Aberrant expression of the glycolytic enzymes aldolase B and type II hexokinase in hepatocellular carcinoma are predictive markers for advanced stage, early recurrence and poor prognosis. Oncol Rep 19(4): 1045-1053.

Pietsch T, Gottert E, Meese E, Blin N, Feickert HJ, Riehm H, Kovacs G (1988) Characterization of a continuous cell line (MHH-NB-11) derived from advanced neuroblastoma. Anticancer Res 8(6): 1329-1333.

Polakis PG, Wilson JE (1982) Purification of highly bindable rat brain hexokinase by high performance liquid chromatography (HPLC). Biochem Biophys Res Commun 107(3): 937-943.

Pradelli LA, Beneteau M, Chauvin C, Jacquin MA, Marchetti S, MunozPinedo C, Auberger P, Pende M, Ricci JE (2010) Glycolysis inhibition sensitizes tumor cells to death receptors-induced apoptosis by AMP kinase activation leading to Mcl-1 block in translation. Oncogene 29(11): $1641-1652$.

Qin JZ, Xin H, Nickoloff BJ (2010) 3-Bromopyruvate induces necrotic cell death in sensitive melanoma cell lines. Biochem Biophys Res Commun 396(2): 495-500.

Sanchez-Arago M, Cuezva JM (2011) The bioenergetic signature of isogenic colon cancer cells predicts the cell death response to treatment with 3-bromopyruvate, iodoacetate or 5-fluorouracil. J Transl Med 9: 19.

Sethi N, Kang Y (2011) Unravelling the complexity of metastasis - molecular understanding and targeted therapies. Nat Rev Cancer 11(10): 735-748.

Sheng H, Niu B, Sun H (2009) Metabolic targeting of cancers: from molecular mechanisms to therapeutic strategies. Curr Med Chem 16(13): 1561-1587.

Stubbs M, Bashford CL, Griffiths JR (2003) Understanding the tumor metabolic phenotype in the genomic era. Curr Mol Med 3(1): 49-59.

Subarsky P, Hill RP (2003) The hypoxic tumour microenvironment and metastatic progression. Clin Exp Metastasis 20(3): 237-250.

Vali M, Liapi E, Kowalski J, Hong K, Khwaja A, Torbenson MS, Georgiades C, Geschwind JFH (2007) Intraarterial therapy with a new potent inhibitor of tumor metabolism (3-bromopyruvate): Identification of therapeutic dose and method of injection in an animal model of liver cancer. $J$ Vasc Interv Radiol 18(1): 95-102.

Warburg O (1956) On the origin of cancer cells. Science 123(3191): 309-314.

This work is published under the standard license to publish agreement. After 12 months the work will become freely available and the license terms will switch to a Creative Commons AttributionNonCommercial-Share Alike 4.0 Unported License. 